\title{
Interactive Lighting Design for Multi-device Virtual Environments
}

\author{
Man Lok Yau, Jason Moore, Bill Tomlinson \\ University of California, Irvine \\ \{mlyau, jzmoore,wmt\}@uci.edu
}

It is increasingly common for multiple computing devices to operate in close proximity. As these devices begin to work together more and more, it may be useful to see a collection of devices as a multi-device system rather than as a group of devices that function separately. One form of multi-device system is the multi-device virtual environment (MDVE), in which several devices work together to create the illusion of a graphical world that stretches seamlessly across their screens. MDVEs can potentially be useful for entertainment, education and industrial applications. There are a number of challenges involved in creating believable and engaging MDVEs and animated agents that exist on them, including areas such as graphics, sound, interaction paradigms and autonomous behavior. This poster presents a technique for improving multi-device virtual environment by enabling the different devices to share the same light source properties, thereby unifying the visual representations on each device.

Any object that blocks light will cast a shadow, and given the shadow of an object, people can determine the source of light by the direction and the length of the shadow. Most graphical applications have virtual light sources that are not affected by the real-world position or orientation of the device on which it is running. This type of arrangement works fine when the device is stationary. For example, when the user is playing a computer game on a desktop computer, the light source in the game can stay stationary and look believable.

However, if the user is controlling a mobile device (e.g. laptop, tablet PC, PDA), virtual lighting is more complex. Since the entire virtual world can be moved by the user, a static virtual light source appears to be moving in the real world. This graphical presentation contradicts what people understand about shadows in real world, which could be solved by a light source that changes over time to match the relative position in the real world. So, for example, if the mobile device can detect its movement and adjust the virtual light source accordingly, it will create an illusion that the light source is maintaining a consistent characteristic in the real world. The goal of this project is to enable multiple devices to maintain a consistent set of real-world lighting conditions, even when some of the devices are being moved by users.

\section{Proposed Implementation}

The proposed implementation is based on the Virtual Raft Project [Tomlinson et al. 2005]. The Virtual Raft Project is an interactive installation that contains three standard desktop computers (islands) and three Toshiba Portege M200 tablet PCs (rafts). The goal of the project is to explore ways to create more intuitive experience for users to interact with virtual characters. The current version of the Virtual Raft Project provides shadows for characters on each of the virtual islands but does not provide shadows for characters on the tablet PCs, as people may be confused about the light source when they move the tablet PC.

The research project currently under way is working to extend the Virtual Raft Project so that the shadows of characters on the tablet PC take physical movements into account. The tablet PCs used in the Virtual Raft Project have built-in accelerometers that can detect motion around the two horizontal axes. The accelerometers are effective for detecting tilting of the device, but they cannot detect rotations around the vertical axis. Therefore, the research team is currently exploring the integration of a digital compass with each mobile device. A digital compass can enable the device to know how it is being rotated and use this information to affect the shadow direction. For example, lets assume the virtual light source is coming from the North in the virtual world. Without the use of digital compass, when the user turns the tablet PC 90 degrees to the right, the virtual light source becomes the East. The shadow of the character seems to have moved 90 degrees in the global perspective and is not consistent in the user's point of view. On the other hand, a tablet PC with a digital compass could detect the angle of the turn and notify the software to move the virtual light source accordingly. It will move the virtual light source 90 degrees to the left to counter the turn made by the user. Therefore, the physical light source appears to be stationary from the perspective of the user. This will lead users to believe that they are actually manipulating the characters, instead of just looking at a display.

A later stage of the project will be creating a model of this physical light source that can be shared between the systems. The current Virtual Raft Project uses infrared (IrDA) for proximity detection and Wireless Ethernet for data transfer. Since IrDA has a limited detection range (both distance and orientation), two devices within IrDA range can share information about the real world location and orientation of the virtual light. This way the devices can constantly update and correct the location of the light source based on the information from the other devices. Through this mechanism, the light source information can be propagated from a device to another.

\section{Summary}

Many graphical applications are interactive in nature and as more people are using mobile devices for these applications, the graphical elements in these applications should take into account the physical movement of the devices. The example in this poster is to use a digital compass to detect turnings of a tablet PC and adjust the light source in the virtual world, and also to enable multiple devices to share a common lighting scheme by propagating it via IrDA communication. These two elements will create an illusion for the user that the physical light source stays the same and matches their experience with real world shadows. By creating coherent lighting designs across multiple collocated devices, this technique seeks to improve the believability and engagement of interactive graphical applications.

\section{References}

TOMLinson, B., YAU, M. L., O'CONNELl, J., Williams, K. and YAMAOKA, S., 2005. The Virtual Raft Project: A Mobile Interface for Interacting with Communities of Autonomous Characters, In Conference Abstracts and Applications, ACM Conference On Human Factors In Computing Systems (CHI 2005). 